\title{
Excitotoxic Lesions of the Amygdala Fail to Produce Impairment in Visual Learning for Auditory Secondary Reinforcement But Interfere with Reinforcer Devaluation Effects in Rhesus Monkeys
}

\author{
Ludiše Málková, ${ }^{1}$ David Gaffan, ${ }^{2}$ and Elisabeth A. Murray ${ }^{1}$ \\ ${ }^{1}$ Laboratory of Neuropsychology, National Institute of Mental Health, Bethesda, Maryland 20892, and 2Department of \\ Experimental Psychology, Oxford University, Oxford OX1 3UD, England, United Kingdom
}

\begin{abstract}
Aspiration lesions of the amygdala were found previously to produce a severe impairment in visual discrimination learning for auditory secondary reinforcement in rhesus monkeys (Gaffan and Harrison, 1987). To determine whether excitotoxic amygdala lesions would also produce this effect, we trained four naive rhesus monkeys on the same task. The monkeys were required to learn 40 new visual discrimination problems per session in a situation in which visual choices were guided by an auditory secondary reinforcer that had been previously associated with food reward. Bilateral excitotoxic lesions of the amygdala had no effect on the rate of learning visual discrimination problems for auditory secondary reinforcement. We also tested the amygdalectomized monkeys on a reinforcer devaluation task and compared their performance with a group of three normal monkeys. The monkeys first learned to discrimi-
\end{abstract}

nate 60 pairs of objects, baited with two different food rewards. Each of the food rewards was then devalued by selective satiation in two separate experimental sessions. Normal controls tended to avoid displacing objects that covered the devalued food to a significantly greater degree than did the amygdalectomized monkeys, indicating that the excitotoxic amygdala damage interfered with reinforcer devaluation effects. Our results are consistent with the idea that the amygdala is necessary for learning the association between stimuli and the value of particular food rewards; however, the amygdala is not necessary for maintaining the value of secondary reinforcers, once they have been learned.

Key words: amygdala; ibotenic acid; visual discrimination; auditory; secondary reinforcement; reinforcer devaluation; rhesus monkey
Visual discrimination learning for food reward is a complex task, one usually thought to tax stimulus-reward association. Although the amygdala is clearly implicated in certain aspects of visual discrimination learning for food reward in monkeys, the effects of bilateral amygdala removals on visual discrimination learning in a manual test apparatus can be quite variable, ranging from no impairment (Schwartzbaum, 1965; Horel et al., 1975) to mild (Malamut et al., 1984) or severe impairment (Schwartzbaum and Poulos, 1965; Spiegler and Mishkin, 1981). More consistent effects on visual discrimination learning after aspiration lesions of the amygdala are evident in automated apparatuses (Gaffan and Murray, 1990; Gaffan, 1994).

To analyze the manner in which the amygdala contributed to visual discrimination learning, Gaffan and Harrison (1987) designed a task that used secondary reinforcement and a different sensory modality for the discriminanda (visual) and the secondary reinforcer (auditory). In their task, a monkey had to solve a new set of visual discrimination problems in every experimental session. The only information concerning correct or incorrect

\footnotetext{
Received Feb. 4, 1997; revised April 30, 1997; accepted May 22, 1997.

This research was supported by the Human Frontiers Scientific Program Organization (E.A.M.) and the National Institute of Mental Health Intramural Research Program. We thank P. Holland and M. Mishkin for valuable comments on an earlier version of this manuscript, W. Hadfield for technical assistance, J. N. Sewell for help with histological processing of the tissue, and the staff of the In Vivo Nuclear Magnetic Resonance Research Center for performing the MRI scans. We thank the United Kingdom Medical Research Council's Interdisciplinary Research Centre at Oxford University for a travel grant.

Correspondence should be addressed to Dr. Elisabeth A. Murray, Laboratory of Neuropsychology, National Institute of Mental Health, National Institutes of Health, Building 49, Room 1B80, Bethesda, MD 20892.

Copyright (C) 1997 Society for Neuroscience $0270-6474 / 97 / 176011-10 \$ 05.00 / 0$
}

choices in each problem was the presentation of one of two auditory stimuli that had previously been associated with either food reward (the auditory reinforcer) or no food reward (the nonreinforcer). The monkey had to choose, based only on the auditory feedback, the correct stimulus four times in a row to obtain a food reward. Using this task, Gaffan and Harrison (1987) showed that bilateral aspiration lesions of the amygdala severely impaired visual discrimination learning for auditory secondary reinforcement in rhesus monkeys. Furthermore, disconnection of the amygdala from auditory sensory cortex, but not its disconnection from visual association cortex, also disrupted learning. The authors concluded that the amygdala is important for maintaining the association of the auditory secondary reinforcer with the intrinsic incentive value of the food reward.

Recent studies have demonstrated that selective amygdala lesions can yield different behavioral effects than aspiration lesions of the amygdala in monkeys (O’Boyle et al., 1993; Málková and Murray, 1996; Murray et al., 1996). One possible explanation for this difference is that aspiration lesions of the amygdala typically include not only the amygdaloid complex but also the adjacent entorhinal, piriform, and periamygdaloid cortex. In addition, it is likely that efferent fibers of the perirhinal cortex coursing just lateral to the amygdala are transected, thereby rendering the anterior portion of the perirhinal cortex dysfunctional as well (Murray, 1992; Goulet et al., 1996).

To test whether the finding of Gaffan and Harrison (1987) would still hold for lesions limited to the neurons of the amygdala, we examined the effects of excitotoxic amygdala lesions on their task (Experiment 1). If excitotoxic amygdala lesions yielded an impairment, then the conclusion that the amygdala is necessary 
for maintaining the value of a secondary reinforcer would be reconfirmed. If excitotoxic amygdala lesions failed to yield an impairment, then some structure(s) other than the neurons of the amygdala, either alone or in combination with the amygdalar neurons, must be critical for efficient learning of the task.

\section{EXPERIMENT 1}

\section{Materials and Methods}

\section{Subjects}

Four experimentally naive rhesus monkeys (Macaca mulatta), one female and three males, were used. They weighed $3.5-5.0 \mathrm{~kg}$ at the beginning of the study, were housed individually in rooms with automatically regulated lighting (12 hr light/dark cycle), and were maintained on primate chow (no. 5038, PMI Feeds, St. Louis, MO) supplemented with fresh fruit. Water was always available in the home cage.

\section{Apparatus and materials}

The monkeys were trained in an automated apparatus consisting of an IBM computer connected to a color monitor fitted with a touch-sensitive screen (Microtouch Systems, Woburn, MA), an automated pellet dispenser (BRS/LVE, Laurel, MD), and a loudspeaker.

A large set of visual stimuli was used. Each stimulus consisted of two different ASCII characters of two different colors and two different sizes superimposed. These stimuli were created by an algorithm described previously (Murray et al., 1993). The auditory stimuli were digitized sounds corresponding to "Wail" and "A440," which were selected because monkeys in an earlier study (Gaffan and Harrison, 1991) found them to be more easily discriminable than other sounds. One stimulus was assigned as a secondary reinforcer (i.e., associated with food reward) and the other as a secondary nonreinforcer (nonrewarded) for two monkeys, and this assignment was reversed for the remaining two monkeys.

For each test session, the monkey was seated in a primate chair inside a testing cubicle. The monkey's head was $\sim 230 \mathrm{~mm}$ from the monitor, and the monkey's arms were free to reach toward any part of the screen. A visual stimulus could appear in one of two positions on the monitor, either on the left or the right side of the screen, $90 \mathrm{~mm}$ from the center. The auditory stimuli were played through a loudspeaker that was located on the floor of the cubicle below the center of the monitor. A food cup, which received rewards through a tube connected to the pellet dispenser, was located directly below the center of the monitor. The food rewards were banana-flavored pellets $(190 \mathrm{mg})$ (Noyes, Lancaster, NH). Infrared light sources and an infrared sensitive closed-circuit television camera enabled the monkey to be observed by the experimenter during the test sessions.

\section{Preoperative testing}

The monkeys were trained in a series of stages, described below, with the aim of having them learn to solve visual discrimination problems on the basis of auditory secondary reinforcement.

Pretraining. The preliminary training was performed in two stages. In the first stage, the monkey learned to touch a stimulus that appeared on the monitor screen to receive a reward. A single stimulus appeared on either the left or the right side of the monitor screen. If the monkey touched the stimulus, the auditory secondary reinforcer was presented together with the visual stimulus for $1 \mathrm{sec}$, and both were then terminated simultaneously. Two banana pellets were delivered. If the monkey did not touch the stimulus within $30 \mathrm{sec}$, then the auditory reinforcer was presented at the end of the $30 \mathrm{sec}$ interval, the visual and the auditory stimuli were terminated simultaneously, and two pellets were delivered. Thirty novel stimuli were randomly presented based on a 2 min variable interval. The criterion for completion of this stage was 2 consecutive $d$ with one or more responses.

The main purpose of the second stage of pretraining was to familiarize the monkey with the multiple responses that would be required for reward delivery in the main task. As in the first stage, a single visual stimulus appeared on either the left or the right side of the monitor screen. Now, however, reward delivery was contingent on the monkey's response. When the monkey touched the visual stimulus, the auditory secondary reinforcer was presented for $1 \mathrm{sec}$, and then both the auditory and visual stimuli were immediately terminated. At first, only a single touch to the visual stimulus led to delivery of food reward. In later sessions, however, two to four touches, on consecutive trials, were re- quired to obtain food reward. In the final step, the monkey was required to touch the visual stimulus four times consecutively, one touch per trial, to obtain the two-pellet reward. Forty different "problems" were presented in each session. The interval between presentations of different problems was $13 \mathrm{sec}$, and the interval between trial presentations within problems was $3.5 \mathrm{sec}$.

Main task. In the main task, each monkey was required to solve new visual discrimination problems when the only feedback provided was the occurrence of the auditory secondary reinforcer or nonreinforcer. On each trial, two visual stimuli were presented simultaneously on the screen, one arbitrarily designated positive, the other negative. If the monkey touched the positive stimulus, the auditory reinforcer was presented together with both the visual discriminanda for $1 \mathrm{sec}$, and all stimuli were terminated simultaneously. If the monkey touched the negative stimulus, the auditory nonreinforcer was presented together with both the visual discriminanda for $1 \mathrm{sec}$, and again, all terminated simultaneously. The same problem was presented over and over again, with left and right positions of the stimuli following a random order, until the monkey solved or lost the problem. At the final stage of the task, a problem was either solved or lost if either the positive or the negative stimulus was chosen on four consecutive trials, respectively. Another problem, composed of two novel stimuli, was then presented. When a problem was solved, the food reward was delivered; when a problem was lost, no food was delivered. Either one of these two possibilities terminated the problem. Thus the number of trials per problem was not fixed but was always at least four, enabling learning curves to be drawn for trials $1-4$. As in the pretraining phase, the intertrial interval between problems was $13 \mathrm{sec}$, and the interval between trials within a problem was $3.5 \mathrm{sec}$.

The criterion for the main task was to finish a session and to solve $90 \%$ or more of the problems $3 \mathrm{~d}$ in a row. On reaching this criterion, each monkey was given 20 daily sessions, which served as the preoperative baseline.

\section{Postoperative testing}

Sixteen to eighteen days after surgery, postoperative testing was initiated. Each monkey received 20 daily sessions of the main task, which were administered in the same way as before surgery.

\section{Surgery}

Before surgery each monkey was anesthetized, placed in a specially constructed nonferrous stereotaxic frame, and given a brain scan with the magnetic resonance imaging (MRI) technique. The MRI scans were used to obtain measurements of the amygdala relative to both the interaural plane (earbars) and the midline, which were also visible on the scan (Saunders et al., 1991). On the basis of these measurements, stereotaxic coordinates for a matrix of injection sites in the amygdala were determined. The sites were separated by $\sim 2 \mathrm{~mm}$ in each plane and were intended, on the basis of empirical findings, to allow diffusion of the excitotoxin ibotenic acid throughout the entire amygdala. All four monkeys received injections of ibotenic acid (Regis Chemical, Morton Grove, IL) in a two-stage surgery after the procedure described by Murray et al. (1996). Because a large and potentially lethal (toxic) amount of ibotenate would have been required to produce an amygdala lesion in both hemispheres in a single stage of surgery, and because the slow injection rate also precluded making a bilateral lesion in a single stage, the monkeys received the amygdala lesions in two stages, left hemisphere followed by right, separated by a minimum of 2 weeks (range, 14-21 d). There was no interoperative testing.

At the time of surgery, each monkey was anesthetized and placed in the stereotaxic frame. The anesthesia was induced with ketamine hydrochloride $(10 \mathrm{mg} / \mathrm{kg})$ and maintained under isoflurane gas $(1.0-2.0 \%, \mathrm{v} / \mathrm{v}$, to effect) for the duration of the surgery, which was performed under aseptic conditions. Monkeys received an intravenous drip solution of isotonic fluids, and heart rate, respiration rate, blood pressure, expired $\mathrm{CO}_{2}$, and body temperature were monitored throughout the procedure. A bone flap was made in the appropriate portion of the cranium, and small slits were cut in the dura to allow the needle of a $10 \mu \mathrm{l}$ Hamilton syringe, held in a Kopf electrode manipulator (David Kopf Instruments, Tujunga, CA), to be lowered to the proper coordinates. The monkeys received 16-27 injections per hemisphere via a 30 gauge needle. A total of $1 \mu \mathrm{l}$ of ibotenic acid $(10-15 \mathrm{mg} / \mathrm{ml})$ was injected at each site. To allow diff usion of the ibotenic acid into the extracellular space, and to minimize mechanical damage to the tissue, all injections were made at a rate of $0.2 \mu \mathrm{l} / \mathrm{min}$. After the injections were completed, the scalp was closed in anatomical layers. All 
Table 1. Percent damage

\begin{tabular}{|c|c|c|c|c|c|c|c|c|c|}
\hline \multirow[b]{2}{*}{ Subject } & \multicolumn{3}{|c|}{ Amygdala } & \multicolumn{3}{|c|}{ Entorhinal cortex } & \multicolumn{3}{|c|}{ Perirhinal cortex } \\
\hline & $\mathrm{L}$ & $\mathrm{R}$ & Mean & $\mathrm{L}$ & $\mathrm{R}$ & Mean & $\mathrm{L}$ & $\mathrm{R}$ & Mean \\
\hline A1 & 92 & 50 & 71 & 4 & 0 & 2 & 2 & 0 & 1 \\
\hline A2 & 54 & 93 & 74 & 0 & 1 & 0 & 0 & 0 & 0 \\
\hline A3 & 100 & 96 & 98 & 42 & 8 & 25 & 6 & 1 & 4 \\
\hline A4 & 93 & 97 & 95 & 3 & 5 & 4 & 0 & 0 & 0 \\
\hline
\end{tabular}

Numerals indicate percent damage of left (L), right (R), and total (mean) volume for each of three structures: amygdala, entorhinal cortex, and perirhinal cortex. A1-A4 are monkeys with excitotoxic amygdala lesions.

[De A1 VIIA Case A4
Intended Lesion
Case A2

U1IA Case A3
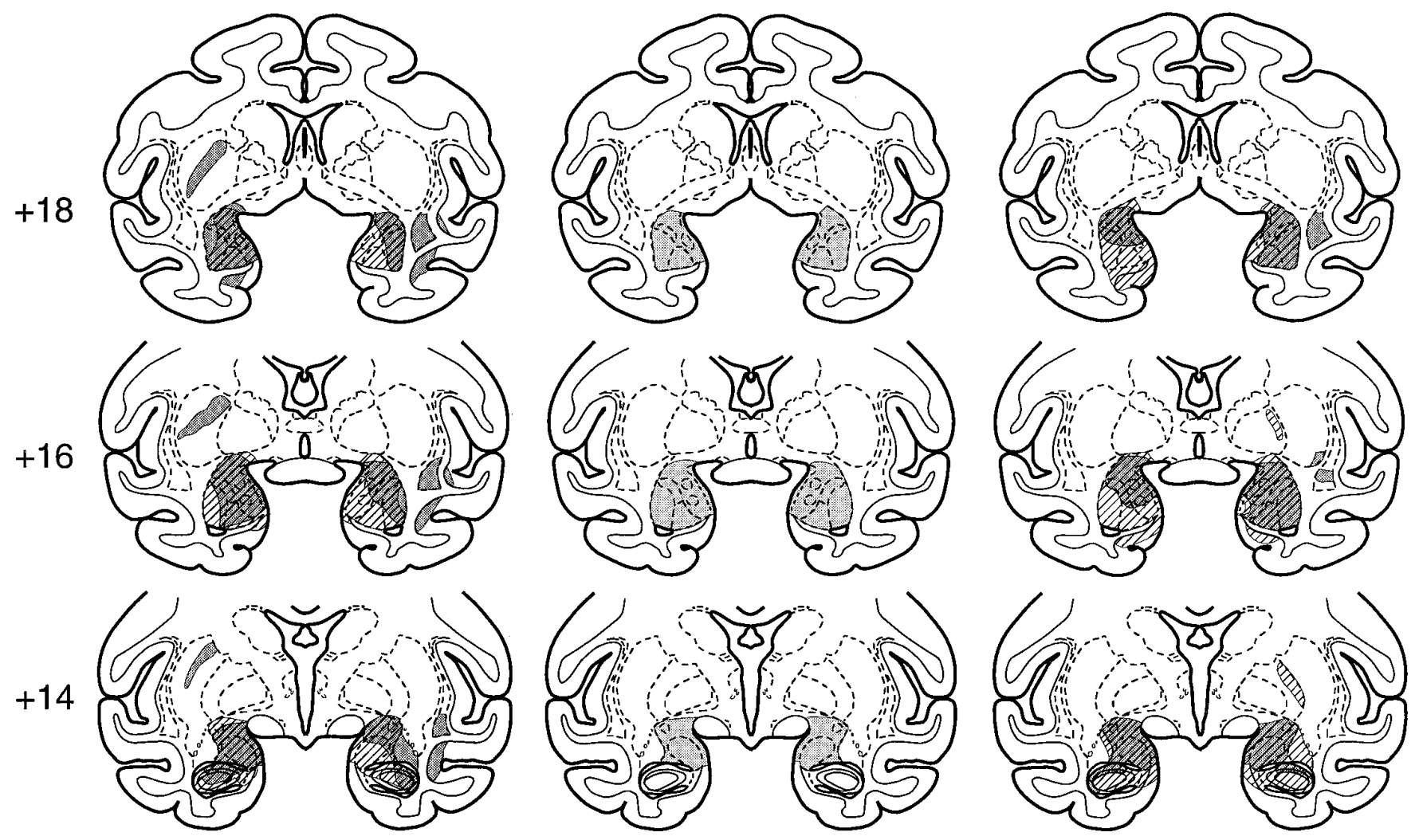

Figure 1. Coronal sections showing the intended lesion (middle column) and the actual extent of damage in four monkeys, A1 and A4 (left column) and A 2 and A3 (right column), that received injections of ibotenic acid into the amygdala. Numerals indicate the distance in millimeters from the interaural plane.

monkeys received a pre- and postoperative treatment regimen consisting of dexamethasone sodium phosphate $(0.4 \mathrm{mg} / \mathrm{kg})$ and Di-Trim $(0.1 \mathrm{ml} / \mathrm{kg}$, $24 \%$ w/v solution, i.m.) (Syntex Animal Health, West Des Moines, IA) for $1 \mathrm{~d}$ before surgery and 1 week after surgery to reduce swelling and to prevent infection, respectively. They also received acetaminophen $(40 \mathrm{mg})$ for $3 \mathrm{~d}$ after surgery for relief of pain.

\section{Histology}

On completion of the experiment, the monkeys were given an overdose of barbiturates (sodium pentobarbital, $100 \mathrm{mg} / \mathrm{kg}$, i.p.) and were perfused through the heart with normal saline followed by aldehyde fixatives. The brains were removed, photographed, and frozen. Tissue was sectioned at 50 $\mu \mathrm{m}$ on a freezing microtome in the coronal plane. Every fifth section was mounted, defatted, stained with thionin, and coverslipped.

The extent of damage to the amygdala in each monkey is indicated in
Table 1 and Figure 1. Representative photomicrographs of the lesion are shown in Figures 2 and 3. The damage was essentially complete in monkeys A3 and A4. In the two remaining monkeys, the damage was nearly complete on either the left (A1) or the right (A2), with less substantial damage $($ mean $=52 \%)$ to the other side.

As for unintended damage, all monkeys sustained slight cell loss in the entorhinal cortex, two monkeys unilaterally (A1 and A2; the damage in A2 appeared only at level +17 and is not shown in Fig. 3) and two bilaterally (A3 and A4). Even in the cases with bilateral involvement, however, the cell loss was restricted mainly to one hemisphere. Furthermore, all monkeys sustained slight cell loss to the portion of the basal nucleus of Meynert dorsally adjacent to the amygdala, two of them (A1 and A4) bilaterally and the other two (A2 and A3) unilaterally, with more cell loss on the right than on the left. In all cases, however, the cell loss was substantially less than in the cases with excitotoxic lesions of the 

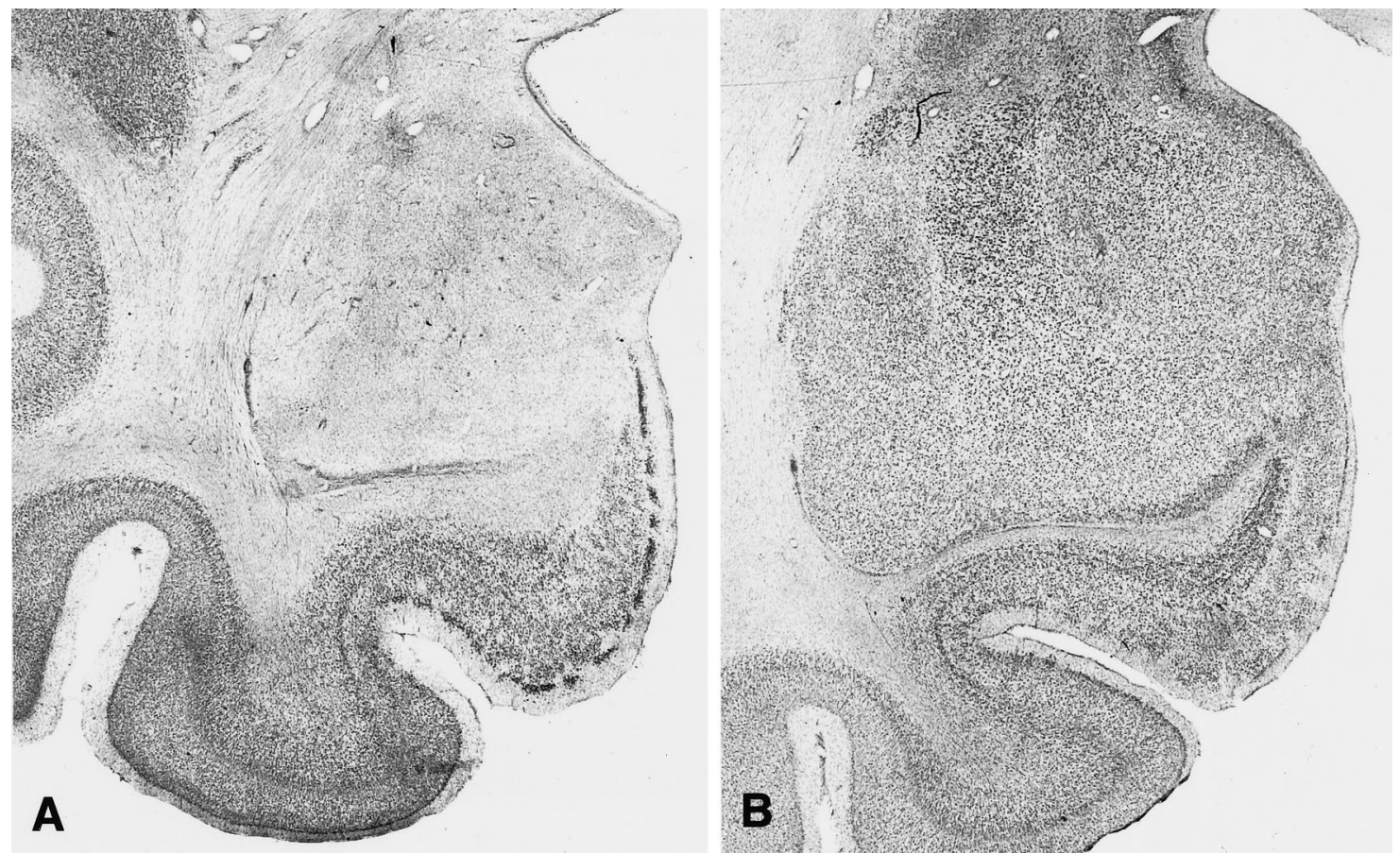

Figure 2. Photomicrographs of Nissl-stained coronal sections approximately $+16 \mathrm{~mm}$ from the interaural plane. $A$, Section through the left amygdala in monkey A4. $B$, Section through the left amygdala in an intact monkey. Note virtually complete cell loss in the amygdala of the operated monkey $(A)$, with relative preservation of the underlying entorhinal cortex.

amygdala reported by Murray et al. (1996). In addition, all monkeys sustained cell loss, $2-4 \mathrm{~mm}$ in anteroposterior extent, in the anterior portion of the hippocampus bilaterally. Additional cell loss was restricted mainly to the CA1 field, extending along the entire length of the hippocampus, bilaterally in A4 and unilaterally in the remaining monkeys. Other unintended damage, sustained unilaterally, included cell loss in the right ventral claustrum (A1 and A2), the fundus of superior temporal sulcus and the insula (A1), and the striatum (A1 and $\mathrm{A} 3$ ), the latter presumably attributable to infarction along the track of the injection needle.

\section{Results}

\section{Preoperative learning and performance}

Three of the four monkeys displayed little difficulty in learning the task. They required a mean of 42 sessions to complete pretraining and attain criterion on the main task. One monkey (A4) failed to attain criterion in approximately 100 sessions; however, because it scored well above chance levels and had attained a stable performance level, the last 15 sessions were considered the preoperative baseline for this monkey.

For monkeys A1, A2, and A3, the final preoperative withinproblem learning curves were based on the last five preoperative sessions, i.e., a total of 200 new problems. For A4, the learning curve was based on the last 15 preoperative sessions (600 new problems). Average error rates for the final problems are presented in Table 2 and Figure 4.

\section{Postoperative performance}

Postoperative error rates are given in Table 2 and Figure 4. The effect of surgery was evaluated in a condition $\times$ trials $(2 \times 4)$ ANOVA with repeated measures. A significant effect of trials $\left[F_{(\text {Greenhouse-Geiser 1,4) }}=32.64 ; p<0.01\right]$ indicated an improvement in performance across the first four trials. The effect of condition $\left[F_{(1,3)}<1 ; p>0.05\right]$ was not significant, nor was the interaction of condition and trials $\left[\mathrm{F}_{(\text {Greenhouse-Geiser 1,4) }}<1 ; p>\right.$
0.05], indicating that there was no difference between preoperative and postoperative performance.

\section{Comment}

There was no effect of bilateral, excitotoxic amygdala lesions on the rate at which monkeys learned visual discriminations for auditory secondary reinforcement. Thus, the impairment seen by Gaffan and Harrison (1987) on the same task after aspiration lesions of the amygdala must have arisen from damage to one or more structures other than the neurons residing in the amygdala. The sites of damage that might have been responsible will be considered in the Discussion.

To determine whether selective amygdala lesions would disrupt learning about food rewards, we tested our amygdalectomized monkeys on a reinforcer devaluation task and compared their performance with that of a group of normal monkeys.

\section{EXPERIMENT 2}

Hatfield et al. (1996) showed that excitotoxic lesions of the basolateral amygdala interfered with reinforcer-devaluation effects in rats. In their classical conditioning paradigm, rats with either basolateral amygdala lesions or sham operations received light-food pairings. One group, comprising roughly half with amygdala lesions and half controls, received two pairings of food pellets with an injection of $\mathrm{LiCl}$, a toxin that produces malaise, which was intended to devalue the food pellets. A second group, also comprising roughly half with amygdala lesions and half controls, received the food pellets and $\mathrm{LiCl}$ unpaired, which was expected to leave the value of food pellets intact. Indeed, the food pellet consumption decreased in all the devalued subjects (pellets and $\mathrm{LiCl}$ injections paired), regardless of lesion status, but did not change in the nondevalued (unpaired) groups. Subsequently, conditioned reactions to the light were examined in the absence of 

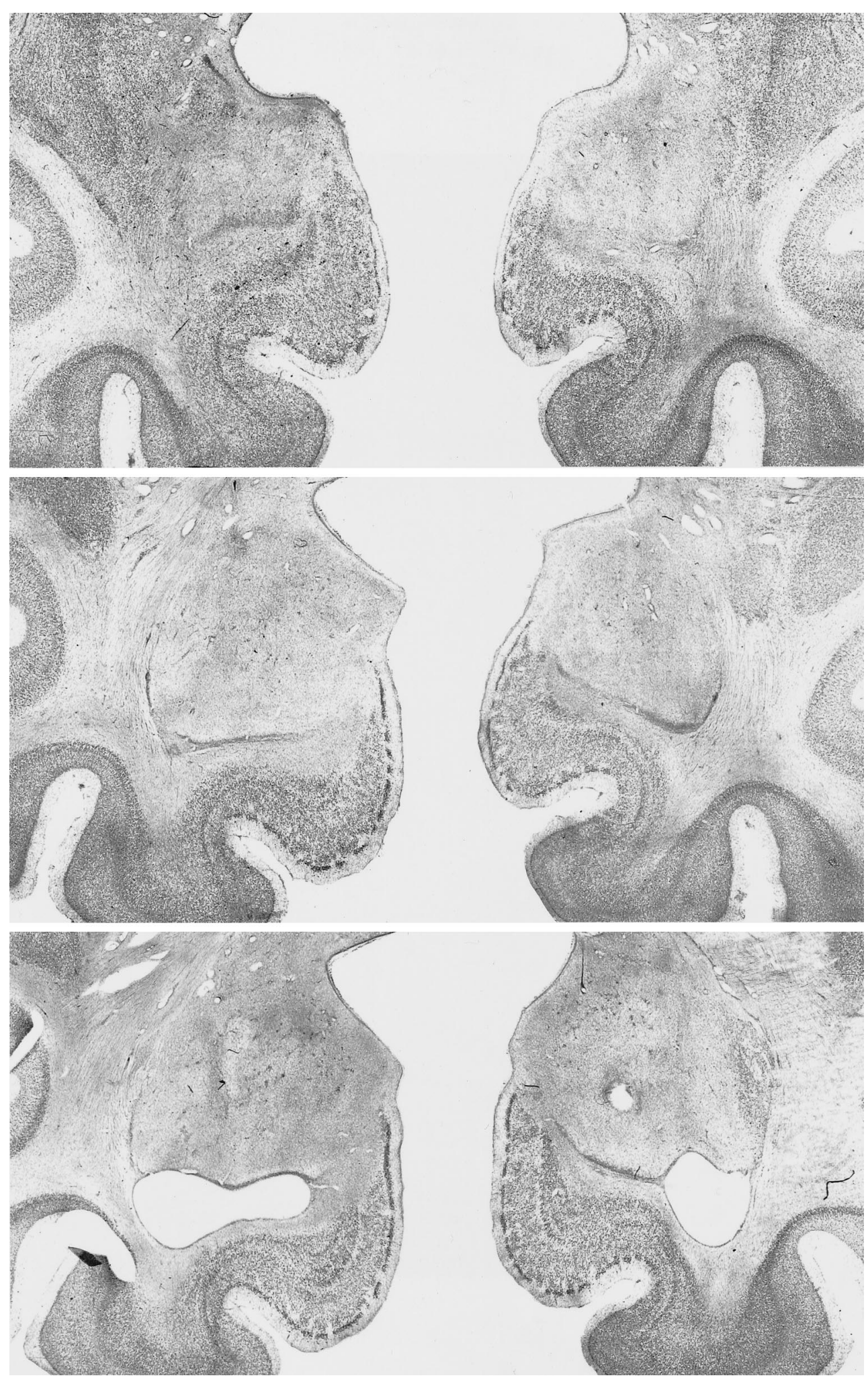

Figure 3. Photomicrographs of Nisslstained coronal sections through the amygdala lesion in monkey A4. Top, middle, and bottom sections represent the left and right amygdala at approximately +18 , +16 , and $+14 \mathrm{~mm}$ from the interaural plane, respectively. food presentations. As expected, the nondevalued subjects did not alter their behavior, i.e., they maintained conditioned responses to the light. By contrast, in the devalued subjects, the responses to the light were significantly reduced in intact rats, whereas rats with basolateral amygdala lesions maintained their levels of responding. The authors thus confirmed a previous result (Holland and Straub, 1979) showing a drop in conditioned reactions after the uncondi- tioned reinforcer (food) was devalued by administration of $\mathrm{LiCl}$, and in addition they showed that the devaluation effect is critically dependent on the basolateral amygdala.

We devised a reinforcer-devaluation procedure for monkeys that used operant conditioning methods to test whether the amygdala is essential for devaluation effects in monkeys as well as in rats. Our devaluation procedure was based on selective satiation, 
Table 2. Percent error in learning visual discrimination problems for auditory secondary reinforcement

\begin{tabular}{|c|c|c|c|c|c|c|c|c|}
\hline & \multicolumn{4}{|c|}{ Preoperative learning trials } & \multicolumn{4}{|c|}{ Postoperative learning trials } \\
\hline & 1 & 2 & 3 & 4 & 1 & 2 & 3 & 4 \\
\hline $\mathrm{A} 1$ & 49.0 & 10.5 & 6.5 & 2.5 & 39.5 & 5.5 & 2.0 & 1.0 \\
\hline $\mathrm{A} 2$ & 46.5 & 15.5 & 11.5 & 8.5 & 39.0 & 24.0 & 15.0 & 10.5 \\
\hline A3 & 43.0 & 30.5 & 17.0 & 10.0 & 54.5 & 28.0 & 24.0 & 12.0 \\
\hline $\mathrm{A} 4^{a}$ & 47.2 & 37.5 & 26.2 & 19.2 & 46.0 & 40.5 & 32.5 & 25.5 \\
\hline Mean & 46.4 & 23.5 & 15.3 & 10.1 & 44.8 & 24.5 & 18.4 & 12.3 \\
\hline
\end{tabular}

Numerals indicate the mean percent error obtained on trials 1-4 for problems learned in the last 5 of 20 sessions administered before surgery (preoperative learning) and the last 5 of 20 sessions administered after surgery (postoperative learning). A1-A4 are monkeys with excitotoxic amygdala lesions.

${ }^{a}$ Did not reach criterion. Used mean score for last 15 sessions before operation.

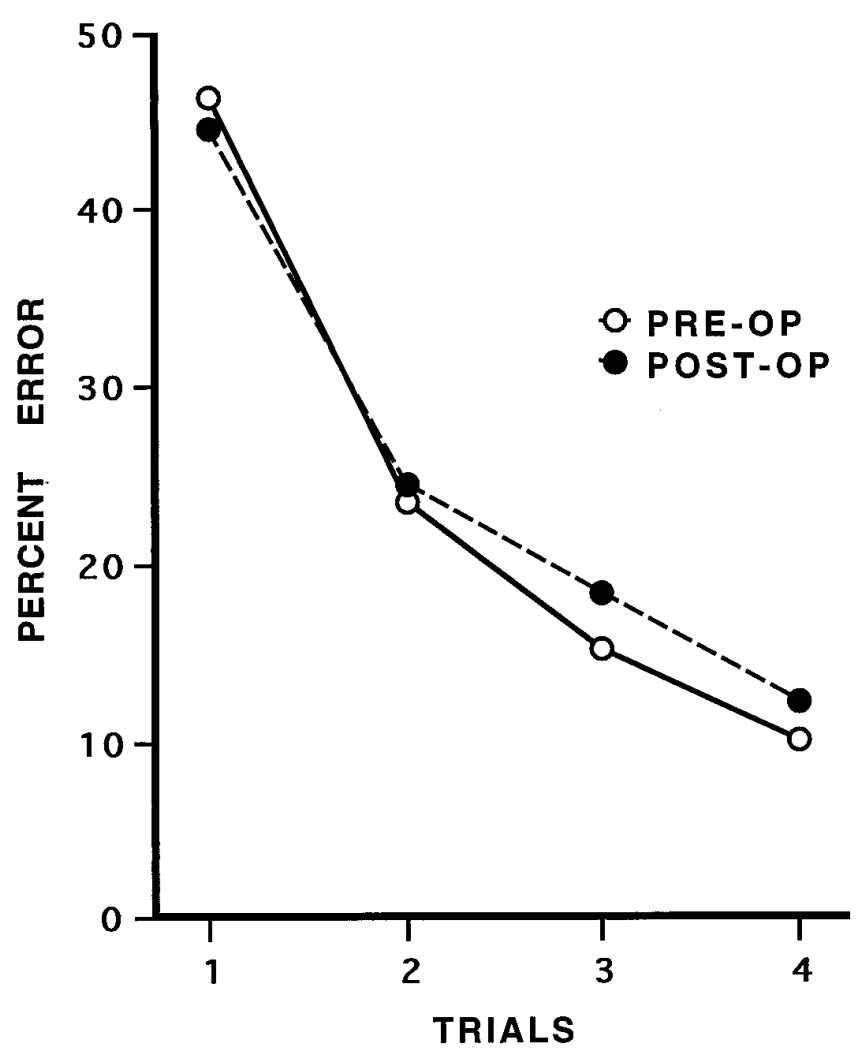

Figure 4. Within-problem learning curves on visual discrimination for auditory secondary reinforcement (Experiment 1$)$ before $(P R E-O P)$ and after $(P O S T-O P)$ excitotoxic amygdala lesions. Average percent error is shown for the first four trials of new problems (see Table 1).

which represents a motivational manipulation (unconditioned) as compared with an associative manipulation (conditioned) used by Hatfield et al. (1996).

\section{Materials and Methods \\ Subjects}

All four monkeys from Experiment 1 were used. In addition, three normal male rhesus monkeys, controls in a separate experiment, served as unoperated controls. They weighed $7.10-9.30 \mathrm{~kg}$ at the beginning of the study. Before the present study, all three had been trained on visual delayed nonmatching-to-sample, and two of them (C1 and $\mathrm{C} 2)$ had received additional training on visual object discriminations. The monkeys were housed and fed in the same manner as that described in Experiment 1.

\section{Apparatus and materials}

The monkeys were trained in a Wisconsin General Testing Apparatus located in a darkened room. The test compartment was illuminated with two $60 \mathrm{~W}$ incandescent bulbs, but the monkey's compartment was always unlit. Extraneous sound masking was provided by a white-noise generator. The test tray, which was located at the level of the floor of the monkey's transport cage, contained two food wells spaced $290 \mathrm{~mm}$ apart, center to center, on the midline of the tray. The wells were $38 \mathrm{~mm}$ in diameter and $6 \mathrm{~mm}$ deep. The stimuli were 120 junk objects that differed widely in shape, size, color, and texture. There were two different food rewards. One was a single "fruit snack," a chewy candy about $1 \mathrm{~cm}$ in size made from fruit juice (Fruit Snacks, Super Giant, Inc.), and the other was a half peanut. In a pilot experiment conducted with other monkeys, these two food rewards were found to be equally palatable.

\section{Testing procedure}

Stage 1. Visual discrimination learning. Monkeys were first trained to discriminate a set of 60 pairs of objects. For each pair, one object was arbitrarily designated positive (i.e., baited with a food reward) and the other negative (i.e., unbaited). On each trial, the two objects comprising a pair, one positive and one negative, were presented for choice, each overlying one of the two food wells on the test tray. Each pair of objects appeared in only one trial per session. Thus, each of the 60 pairs was presented once per session, yielding a total of 60 trials per day. Half of the positive objects were randomly assigned to be baited with a fruit snack (Food-1 objects), the other half with the peanut reward (Food-2 objects). The positive and negative objects within each pair, the food reward assignment, and the order of the pairs remained constant across sessions, but the left-right position of the positive objects in each pair followed a pseudorandom order. The intertrial interval was 20 sec. Criterion was set at a mean of $90 \%$ correct responses over 5 consecutive d (i.e., 270 correct responses out of 300 ).

Stage 2. Reinforcer devaluation. After they attained criterion on Stage 1, the monkeys' choices of objects were assessed in four critical test sessions, each performed on a separate day. In these sessions, only the positive objects were used; the negative objects were set aside to be used in the regular training sessions that intervened between critical test sessions (see below). Thirty pairs, each consisting of one Food-1 and one Food-2 object, were randomly generated before each critical test session. During critical sessions, both objects were baited with the appropriate food on all trials. Each of the pairs was presented once, yielding 30 trials per session. The monkeys were allowed to choose one of the objects in each pair and to obtain the reward. Our measure was the number of Food-1 and Food-2 objects chosen in each session. Two of the four critical test sessions were preceded by a selective satiation procedure, described below, one for each food. The other two were preceded by no satiation procedure and therefore served as the baseline. Between critical sessions, the monkeys were given one regular training session with the original set of 60 object discrimination problems presented for choice in the same manner as during original learning. In addition, at least $2 \mathrm{~d}$ of rest followed each session that had been preceded by the selective-satiation procedure. Critical sessions preceded by satiation occurred in one order (satiation for Food-1, followed by satiation for Food-2) for five monkeys (C2, C3, $\mathrm{A} 1, \mathrm{~A} 3$, and A4), and in the reverse order for two monkeys (C1, A2). In addition, the critical sessions preceded by no satiation (baseline) were administered in two different orders: the first baseline session preceded the first selective satiation session and the second followed the second 
Table 3. Reinforcer devaluation by selective satiation

\begin{tabular}{|c|c|c|c|c|c|c|c|c|}
\hline & \multicolumn{3}{|c|}{ Baseline } & \multicolumn{5}{|c|}{ Satiation } \\
\hline & 1 & 2 & Mean & Food-1 & Food-2 & & & Dif \\
\hline & F1:F2 & $\mathrm{F} 1: \mathrm{F} 2$ & F1:F2 & $\mathrm{F} 1: \mathrm{F} 2$ & $\mathrm{~F} 1: \mathrm{F} 2$ & DS1 & DS2 & score \\
\hline $\mathrm{C} 1$ & $23: 7$ & $20: 10$ & $21.5: 8.5$ & $10: 20$ & $29: 1$ & 11.5 & 7.5 & 19.0 \\
\hline $\mathrm{C} 2$ & $23: 7$ & $21: 9$ & $22.0: 8.0$ & $6: 24$ & $27: 3$ & 16.0 & 5.0 & 21.0 \\
\hline $\mathrm{C} 3$ & $5: 25$ & $10: 20$ & $7.5: 22.5$ & $1: 29$ & $22: 8$ & 6.5 & 14.5 & 21.0 \\
\hline A1 & $20: 10$ & $19: 11$ & $19.5: 10.5$ & $10: 20$ & $17: 13$ & 9.5 & -2.5 & 7.0 \\
\hline $\mathrm{A} 2$ & $12: 18$ & $16: 14$ & $14.0: 16.0$ & $11: 19$ & $13: 17$ & 3.0 & -1.0 & 2.0 \\
\hline A3 & $11: 19$ & $12: 18$ & $11.5: 18.5$ & $7: 23$ & $15: 15$ & 4.5 & 3.5 & 8.0 \\
\hline A4 & $20: 10$ & $21: 9$ & $20.5: 9.5$ & $16: 14$ & $24: 6$ & 4.5 & 3.5 & 8.0 \\
\hline
\end{tabular}

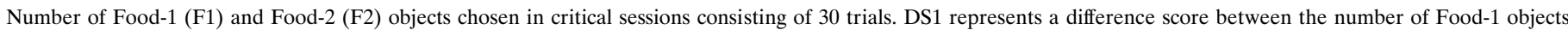

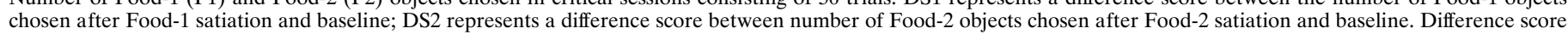
is the sum of DS1 and DS2. C1-C3 are unoperated control monkeys; A1-A4 are monkeys with excitotoxic amygdala lesions.

selective satiation session in four monkeys (C3, A1, A3, and A4), whereas both baseline sessions followed the second selective satiation session in the three remaining monkeys.

Selective satiation procedure. Approximately $24 \mathrm{hr}$ after the last feeding, a food box measuring $8 \times 10 \times 7.5 \mathrm{~cm}$ and attached to the monkey's home cage was filled with $300 \mathrm{gm}$ of peanuts (or $250 \mathrm{gm}$ of fruit snacks) while the monkey was in its home cage. The monkey was allowed to eat the food without being directly observed for $30 \mathrm{~min}$. Then the experimenter entered the room and checked the amount of food eaten. If the monkey had eaten most of the food, an additional $100 \mathrm{gm}$ of peanuts (or $50 \mathrm{gm}$ of fruit snacks) was added to the food box. Whether additional food was given or not, the experimenter started observing the monkey through a window from outside the animal housing room until the monkey refrained from taking food from the food box for $5 \mathrm{~min}$. The test session was then initiated within $\sim 10 \mathrm{~min}$. For the baseline condition, the monkey was simply taken directly from its home cage to the test session without undergoing a selective satiation procedure.

\section{Results}

\section{Stage 1. Visual discrimination learning}

The three unoperated control monkeys required a mean of seven sessions and 142 errors to attain criterion. One of the four monkeys with amygdala lesions (A4) did not reach criterion within 20 sessions; however, its score of $87 \%$ correct responses over the last five sessions was considered sufficiently high to proceed to the next stage. The amygdalectomized monkeys, including A4, required a mean of 11 sessions and 169 errors to attain criterion. The two groups did not differ in their rate of learning the visual discrimination problems [Mann-Whitney $U$ test; $U_{(3,4)}=3.00, p>0.05 ; U_{(3,4)}=4.00, p>0.05$, for the number of sessions and errors, respectively].

\section{Stage 2. Reinforcer devaluation}

In the two baseline sessions (Table 3 ), a group $\times$ session ANOVA with repeated measures showed no difference between the two groups in their choices $\left[F_{(1,5)}<1 ; p>0.05\right]$. In addition, the monkeys' choices of Food-1 versus Food-2 objects remained stable $\left[F_{(1,5)}<1 ; p>0.05\right]$ across the two sessions. Therefore, for the purpose of further analysis, each monkey's baseline was taken to be the mean score for the two sessions. The mean ratio of Food-1:Food- 2 objects selected by the monkeys during the baseline sessions was 17:13 and 16:14 for control and operated monkeys, respectively. There was no significant overall preference for either Food-1 or Food-2 objects within either group [paired $t$ test; $t_{(2)}<1, p>0.05 ; t_{(3)}<1, p>0.05$, respectively].

The amount of food consumed during the selective satiation procedure was compared between groups. The unoperated con-

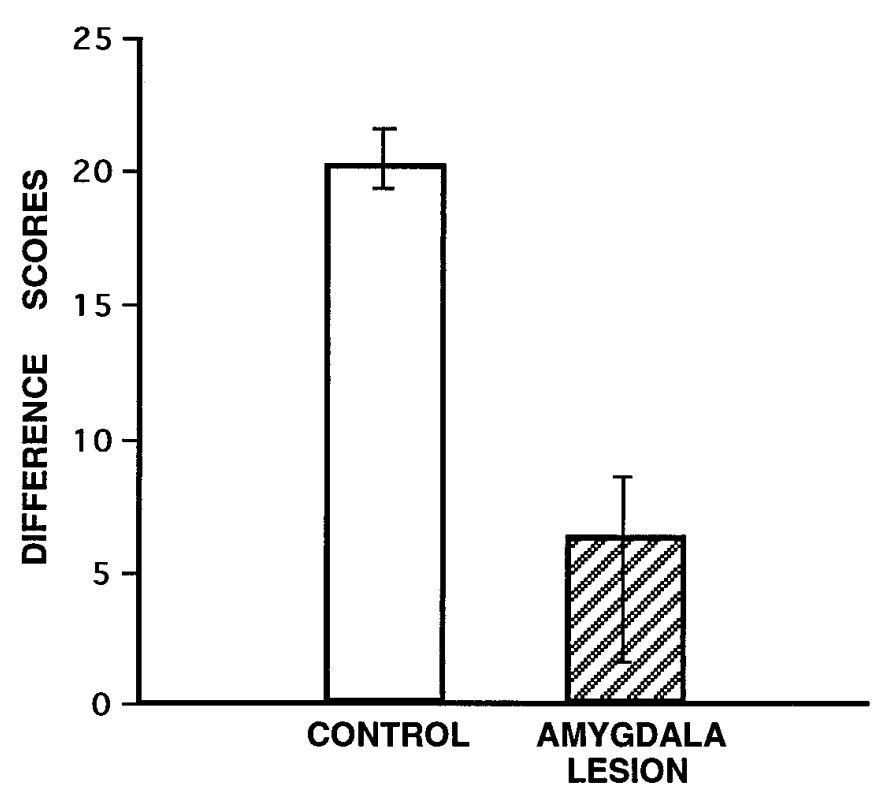

Figure 5. Group mean difference scores, a measure of the effect on choices of objects of reinforcer devaluation (Experiment 2). CONTROL, Unoperated control monkeys $(n=3) ; A M Y G D A L A L E S I O N$, monkeys with selective, excitotoxic lesions of the amygdala $(n=4)$. Vertical lines represent the range of scores of individual monkeys.

trols consumed on average $110 \mathrm{gm}$ of fruit snacks and $250 \mathrm{gm}$ of peanuts, whereas the amygdalectomized monkeys consumed 140 gm and $140 \mathrm{gm}$, respectively. There was no significant difference between the groups on this measure $\left[U_{(3,4)}=4.00, p>0.05 ; U_{(3,4)}\right.$ $=11.0, p>0.05$, for fruit snacks and peanuts, respectively]. Furthermore, both groups spent about the same amount of time in the satiation procedure.

The difference between the number of Food-1 and Food-2 objects chosen in each of the two critical sessions that had been preceded by the selective satiation procedure versus the baseline condition was assessed for each monkey. A positive valence was used for changes in the expected direction. Difference scores from each of the two sessions were summed to obtain a total difference score (Table 3, Fig. 5). For example, monkey C2 chose a mean of 22 Food- 1 and 8 Food- 2 objects in the baseline condition, but only six Food-1 objects after the Food-1 satiation procedure, and only three Food-2 objects after the Food-2 satiation procedure. For 
$\mathrm{C} 2$, the final difference score, a measure of the effect of the selective satiation procedure, was $[(22-6)+(8-3)]$, or 21 . The total difference scores were analyzed by a nonparametric rank sum test [Mann-Whitney $U$ test; $U_{(3,4)}=12.00, p<0.03$ ], which yielded a significant difference between groups. The unoperated controls had higher difference scores than did the monkeys with amygdala lesions. That is, intact monkeys tended to avoid displacing objects that covered the devalued food to a much greater degree than did monkeys with amygdala lesions.

\section{Comment}

Excitotoxic amygdala lesions significantly attenuated the effects of reinforcer devaluation. Although the amygdalectomized monkeys did sustain some inadvertent damage to adjacent structures (e.g., basal forebrain, hippocampus), this damage was variable in extent and typically not bilaterally symmetrical. Thus, it seems highly unlikely that damage outside of the amygdala could account for our result. After being sated with one of the two available food rewards, normal monkeys tended to avoid displacing objects that covered the devalued food in favor of objects overlying the food that had not recently been eaten. By contrast, amygdalectomized monkeys showed little of this tendency. Instead, they displaced about the same number of objects overlying each of the two foods as they had in the baseline (control) sessions. Our results confirm previous findings (Hatfield et al., 1996) that the amygdala plays a necessary role in reinforcer devaluation, and they demonstrate in addition that the effect holds for monkeys as well as for rats and applies to operant conditioning paradigms as well as to classical conditioning paradigms.

There are at least two aspects of the present procedure that are noteworthy. First, to avoid direct associations of the devalued food with a response being measured, critical trials in devaluation studies typically do not involve food reward. In our task, however, we used food rewards to bait all objects on the critical trials. Presumably, this procedure did not affect our ability to discern a devaluation effect, because each trial used a different pair of objects. That is, particular stimulus objects might be directly associated with a devalued food, but only after the objects had already been displaced, and this experience did not seem to affect other trials in the session.

Second, we were surprised by the stability of scores in the baseline sessions. Even though the monkeys received novel (and different) pairings of objects for the two baseline sessions, and even though the selection of objects was different across days, the ratio of Food-1:Food-2 objects chosen by individual monkeys was remarkably stable. Furthermore, some monkeys seemed to exhibit a "food preference," as evidenced by their tendency to select more of the objects that covered one food relative to the other (Table 3). For example, monkeys C1, C2, A1, and A4 consistently displaced more of the Food-1 objects when they were pitted against Food- 2 objects. This phenomenon presumably reflects the different values of the different reinforcers, which operate through a process independent of the amygdala.

\section{DISCUSSION}

Experiment 1 showed that bilateral excitotoxic lesions of the amygdala have no effect on the rate at which monkeys learn visual discriminations for auditory secondary reinforcement. The possibility that the lesions were behaviorally ineffective is ruled out by the positive results of Experiment 2 . There are at least two possible explanations, not necessarily mutually exclusive, of the contrasting effects of aspiration and excitotoxic amygdala lesions. First, the impairments observed by Gaffan and Harrison (1987) could be reinterpreted as a deficit in associating a visual stimulus with the auditory secondary reinforcer. Because at least some types of cross-modal and intramodal stimulus-stimulus associations appear to rely on the integrity of the rhinal cortex (Murray et al., 1993; Murray, 1996), bilateral damage to the rhinal cortex might be responsible for the impairments seen by Gaffan and Harrison (1987). This is possible, because in many cases aspiration lesions of the amygdala include some of the entorhinal cortex, which is removed by direct aspiration, and transect some of the efferent projections of the entorhinal and perirhinal cortex passing by or through the amygdala (Murray, 1992; Goulet et al., 1996), effects which together would be expected to disrupt the function of the rhinal cortex. Furthermore, this proposal is consistent with the findings from the two disconnection groups studied by Gaffan and Harrison (1987). The amygdala-auditory cortex disconnection would be expected to produce a deficit attributable to a failure of auditory information (available in one hemisphere) to gain access to either the ipsilateral rhinal cortex, which is likely damaged in association with the amygdala removal, or the contralateral rhinal cortex, because of transection of much of the anterior commissure through which course the temporal interhemispheric connections. Moreover, the amygdala-visual cortex disconnection would fail to produce a deficit, because although the rhinal cortex is likely damaged because of the amygdala removal in one hemisphere, it is largely intact in the other hemisphere. In this hemisphere, both auditory and visual inputs could still reach the rhinal cortex (despite the visual cortical removal) either directly via the dorsal bank of the superior temporal sulcus (Amaral et al., 1983) or indirectly via the parahippocampal cortex (Martin-Elkins and Horel, 1992; Suzuki and Amaral, 1994). This explanation predicts that a bilateral TE lesion would not impair visual learning for auditory secondary reinforcement, because after a bilateral TE lesion, visual inputs could still reach the rhinal cortex through the superior temporal sulcus and the parahippocampal gyrus.

Second, it is possible that the severe effect of amygdala aspiration on this type of task (Gaffan and Harrison, 1987) was attributable to the interruption of axons passing through the amygdala. These axons would be spared by the excitotoxic lesion that we used in the present study. In a study of taste-aversion learning in the rat, Dunn and Everitt (1988) showed that the impairment produced by an electrolytic lesion of the amygdala was not seen after an excitotoxic lesion, and they showed further that the electrolytic lesion interrupted axons that traveled through the amygdala to the cortex from cells in the brainstem and hypothalamus. They therefore concluded that the effect of the electrolytic amygdala lesion on taste-aversion learning was probably to be ascribed to the interruption of those axons. A similar argument applies to the contrasting effects of aspiration and excitotoxic lesions in monkeys in visual learning for an auditory secondary reinforcer. Thus, recent anatomical data (S. A. Gutnikov and D. Gaffan, unpublished observations) show the same pattern of results reported by Dunn and Everitt (1988), that is, retrograde transport from temporal cortex to brain stem and hypothalamus in a normal monkey, but no such transport in a monkey with an aspiration lesion of the amygdala. Furthermore, the cells of the lateral hypothalamus that project through the amygdala in the monkey are in a region where cellular activity is related to primary food reinforcement (Rolls et al., 1976). Therefore, the axons that are interrupted by an aspiration lesion of the amygdala 
could carry information about primary reward to the temporal lobe cortex, where visual and auditory stimuli are analyzed. This explanation of the pattern of results from visual learning for auditory secondary reinforcement is essentially the same as that offered by Gaffan and Harrison (1987), namely that aspiration amygdalectomy impairs the animal's ability to associate visual and auditory stimuli with primary reinforcement. The idea that information about primary reinforcement is encoded in the axons passing through the amygdala, however, is new, the earlier interpretation having been based on the idea that primary reinforcement was encoded by the neurons of the amygdala itself (Gaffan and Harrison, 1987; Gaffan et al., 1988).

In Experiment 2, we found that excitotoxic amygdala lesions did not affect learning of visual object discriminations for primary reinforcement. The same excitotoxic lesions, however, had a profound effect on reinforcer devaluation. Similarly, the results of Hatfield et al. (1996) showed that lesions of the basolateral amygdala in rats spared conditioned light-food reactions but interfered with emotional properties of conditioning, such as the ability of Pavlovian-conditioned stimuli to acquire reinforcing power and to access the current value of the reinforcer after its devaluation. Although both our devaluation procedure and our testing paradigm differed substantially from those used by Hatfield et al. (1996) in rats, the two methods appear to provide effective measures of the same phenomenon: reinforcer devaluation. The involvement of the amygdala in this process is most likely attributable to its role in the association of environmental stimuli, in this case objects, with the value of primary reinforcers, or alternatively, in mediating access of the stimulus representation with the current value of the reinforcer. There is a growing body of evidence that the amygdala and in particular its basolateral complex plays an important role in associative learning processes that connect the representation of a conditioned stimulus with the motivational value of an unconditioned stimulus (Everitt et al., 1991; Hiroi and White, 1991; Everitt and Robbins, 1992; Gallagher and Holland, 1994; Hatfield et al., 1996). Everitt et al. (1991) showed that the connections between the basolateral amygdala and the ventral striatum are critical for the process through which cues acquire reinforcing value. In their study, preference for a distinctive location based on the delivery of a sucrose reinforcer was disrupted by bilateral basolateral amygdala lesions, bilateral ventral striatum lesions, or by disconnection of these two regions by crossed unilateral lesions. This circuit might therefore be important in mediating changes in the emotional significance of reinforcers. Presumably, our amygdalectomized monkeys failed to associate the objects with the hedonic value of the food reward and after devaluation of the reward were unable to adapt their instrumental responses to the altered value of the reinforcer. Apparently, association between the object and the visual properties of the food reward, which would be expected to survive amygdalectomy, were insufficient to effect change in the animal's behavior after the devaluation.

Although the amygdala appears to be necessary for learning the association between stimuli and the value of primary reinforcers (as in Experiment 2), the results of Experiment $1 \mathrm{dem}$ onstrate that the amygdala is not necessary for maintaining the value of secondary reinforcers, once they have been learned. Studies performed with rats suggest that damage to the basolateral amygdala disrupts learning when new secondary reinforcement (Cador et al., 1989; Burns et al., 1993) or new second-order conditioning (Hatfield et al., 1996) is introduced. These findings are consistent with previous evidence that the amygdala is im- portant during the learning of emotionally charged events, but not after the memory has been consolidated (McGaugh et al., 1993; Salinas et al., 1993). For a stimulus to become a secondary reinforcer it must be associated with the primary reinforcing value of food, but it does not need to be associated differentially with one particular foodstuff as opposed to another. For an animal to respond appropriately to our reinforcer devaluation procedure, however, visual discriminative stimuli must be associated in memory with the value of one particular foodstuff so the animal can selectively avoid those objects that cover the devalued foodstuff while choosing those objects that cover the nondevalued foodstuff. Thus, it appears that the cells of the amygdala are necessary for associating stimuli with the value of one particular foodstuff as opposed to the value of another particular foodstuff, but not for associating stimuli with food reward as opposed to no reward.

\section{REFERENCES}

Amaral DG, Insausti R, Cowan WM (1983) Evidence for a direct projection from the superior temporal gyrus to the entorhinal cortex in the monkey. Brain Res 275:263-277.

Burns LH, Robbins TW, Everitt BJ (1993) Differential effects of excitotoxic lesions of the basolateral amygdala, ventral subiculum and medial prefrontal cortex on responding with conditioned reinforcement and locomotor activity potentiated by intra-accumbens infusion of D-amphetamine. Behav Brain Res 55:176-183.

Cador M, Robbins TW, Everitt BJ (1989) Involvement of the amygdala in stimulus-reward associations: interaction with the ventral striatum. Neuroscience 30:77-86.

Dunn LT, Everitt BJ (1988) Double dissociations of the effects of amygdala and insular cortex lesions on conditioned taste aversion, passive avoidance, and neophobia in the rat using the excitotoxin ibotenic acid. Behav Neurosci 102:3-23.

Everitt BJ, Robbins TW (1992) Amygdala-ventral striatal interactions and reward-related processes. In: The amygdala: neurobiological aspects of emotion, memory, and mental dysfunction (Aggleton JP, ed), pp 401-429. New York: Wiley.

Everitt BJ, Morris KA, O’Brien A, Robbins TW (1991) The basolateral amygdala-ventral striatal system and conditioned place preference: further evidence of limbic-striatal interactions underlying rewardrelated processes. Neuroscience 42:1-18.

Gaffan D (1994) Role of the amygdala in picture discrimination learning with $24 \mathrm{hr}$ intertrial intervals. Exp Brain Res 99:411-422.

Gaffan D, Harrison S (1987) Amygdalectomy and disconnection in visual learning for auditory secondary reinforcement by monkeys. J Neurosci 7:2285-2292.

Gaffan D, Harrison S (1991) Auditory-visual associations, hemispheric specialization and temporal-frontal interaction in the rhesus monkey. Brain 114:2133-2144.

Gaffan D, Murray EA (1990) Amygdalar interaction with the mediodorsal nucleus of the thalamus and the ventromedial prefrontal cortex in stimulus-reward associative learning in the monkey. J Neurosci 10:3479-3493.

Gaffan EA, Gaffan D, Harrison S (1988) Disconnection of the amygdala from visual association cortex impairs visual reward-association learning in monkeys. J Neurosci 8:3144-3150.

Gallagher M, Holland PC (1994) The amygdala complex: multiple roles in associative learning and attention. Proc Natl Acad Sci USA 91: 11771-11776.

Goulet S, Dore FY, Murray EA (1996) Aspiration lesions of the amygdala disrupt rhinal cortical efferents to the mediodorsal nucleus of the thalamus in rhesus monkeys. Soc Neurosci Abstr 22:1867.

Hatfield T, Han J-S, Conley M, Gallagher M, Holland P (1996) Neurotoxic lesions of basolateral, but not central, amygdala interfere with Pavlovian second-order conditioning and reinforcer-devaluation effects. J Neurosci 16:5256-5265.

Hiroi N, White NM (1991) The lateral nucleus of the amygdala mediates expression of the amphetamine-produced conditioned place preference. J Neurosci 11:2107-2116.

Holland PC, Straub JJ (1979) Differential effects of two ways of devaluing the unconditioned stimulus. J Exp Psychol 5:65-78. 
Horel JA, Keating EG, Misantone LJ (1975) Partial Kluver-Bucy syndrome produced by destroying temporal neocortex or amygdala. Brain Res 94:347-359.

Malamut B, Saunders RC, Mishkin M (1984) Monkeys with combined amygdalo-hippocampal lesions succeed in object discrimination learning despite $24 \mathrm{hr}$ intertrial intervals. Behav Neurosci 98:770-778.

Málková L, Murray EA (1996) Effects of partial versus complete lesions of the amygdala on cross-modal associations in cynomolgus monkeys. Psychobiology 24:255-264.

Martin-Elkins CL, Horel JA (1992) Cortical afferents to behaviorally defined regions of the inferior temporal and parahippocampal gyri as demonstrated by WGA-HRP. J Comp Neurol 321:177-192.

McGaugh JL, Introini-Colison IB, Cahill LF, Castellano C, Dalmaz C, Parent MB, Williams CL (1993) Neuromodulatory systems and memory storage: role of the amygdala. Behav Brain Res 58:81-90.

Murray EA (1992) Medial temporal lobe structures contributing to recognition memory: the amygdaloid complex versus the rhinal cortex. In: The amygdala: neurobiological aspects of emotion, memory, and mental dysfunction (Aggleton JP, ed), pp 453-470. New York: Wiley.

Murray EA (1996) What have ablation studies told us about the neural substrates of stimulus memory? Semin Neurosci 8:13-22.

Murray EA, Gaffan D, Mishkin M (1993) Neural substrates of visual stimulus-stimulus association in rhesus monkeys. J Neurosci 13: $4549-4561$.

Murray EA, Gaffan EA, Flint Jr RW (1996) Anterior rhinal cortex and amygdala: dissociation of their contributions to memory and food preference in rhesus monkeys. Behav Neurosci 110:30-42.

O' Boyle Jr VJ, Murray EA, Mishkin M (1993) Effects of excitotoxic amygdalo-hippocampal lesions on visual recognition in rhesus monkeys. Soc Neurosci Abstr 19:438.

Rolls ET, Burton MJ, Mora F (1976) Hypothalamic neuronal responses associated with the sight of food. Brain Res 111:53-66.

Salinas JA, Packard MG, McGaugh JL (1993) Amygdala modulates memory for changes in reward magnitude: reversible post-training inactivation with lidocaine attenuates the response to a reduction in reward. Behav Brain Res 59:153-159.

Saunders RC, Aigner TG, Frank JA (1991) Magnetic resonance imaging of the rhesus monkey brain: use for stereotactic surgery. Exp Brain Res 81:443-446.

Schwartzbaum JS (1965) Discrimination behavior after amygdalectomy in monkeys: visual and somaesthetic learning and perceptual capacity. J Comp Physiol Psychol 60:314-319.

Schwartzbaum JS, Poulos DA (1965) Discrimination behavior after amygdalectomy in monkeys: learning set and discrimination reversal. J Comp Physiol Psychol 60:320-328.

Spiegler BJ, Mishkin M (1981) Evidence for the sequential participation of inferior temporal cortex and amygdala in the acquisition of stimulusreward associations. Behav Brain Res 3:303-317.

Suzuki WA, Amaral DG (1994) Perirhinal and parahippocampal cortices of the macaque monkey: cortical afferents. J Comp Neurol 350:497-533. 